\title{
REDES NEURONALES PARA LA OPTIMIZACIÓN DE LA GESTIÓN DEL RIESGO EMPRESARIAL
}

\section{Reiner Solís Villanueva}

\begin{abstract}
Resumen
El presente artículo propone un modelo que contribuya a mejorar los procesos de gestión de riesgos en las empresas del sector construcción, proporcionando un mecanismo para revisar los resultados de evaluación del riesgo de un proyecto, mediante la creación y empleo de una base de datos con indicadores históricos. Se propone el diseño de estos para medir el impacto de los factores de riesgo sobre los objetivos de un proyecto de infraestructura, bajo un esquema de rentabilidad, comparando los datos históricos con los riesgos identificados en el negocio; para tal fin se usaron las redes neuronales artificiales como herramienta de análisis. Asimismo, se revisan las características comunes de las redes neuronales artificiales y se analiza la viabilidad de su aplicación en la optimización del riesgo empresarial en el sector construcción, cuyas empresas han tenido un crecimiento acelerado en los últimos años.
\end{abstract}

Palabras clave: gestión del riesgo / estimación del riesgo / esquema de rentabilidad / factores del riesgo / redes neuronales artificiales. 


\section{Introducción}

El doctor Koprinarov, profesor de Economía en la Universidad de Burgas, Bulgaria, define la gestión del riesgo como un conjunto de actividades que pretenden un doble objetivo: proteger a la empresa y explotar las oportunidades de beneficio que ofrecen los riesgos (2005). En términos generales, define la gestión de riesgo como el arte de oscilar entre la pérdida y el beneficio. En este sentido, la gestión del riesgo actúa en forma dinámica en la empresa, como una parte inherente de su gestión general que analiza y pronostica los eventos de riesgo, y los aborda para mitigarlos y calcular las ventajas de los riesgos aceptables.

El riesgo es la probabilidad de tener éxito o de fracasar cuando se toma la decisión de llevar a cabo un proyecto (Del Carpio y Eyzaguirre 2007); por tal motivo, se hace necesario realizar una evaluación del riesgo que se está asumiendo, escogiendo una métrica adecuada para su evaluación.

Una debilidad de las prácticas existentes en los procesos de gestión del riesgo es la incapacidad para relacionar los riesgos identificados contra el rendimiento de proyectos similares acontecidos en la empresa. Este documento intenta contribuir a mejorar las prácticas actuales de gestión de riesgos en las empresas del sector construcción, diseñando los indicadores de medición de los factores de riesgo que impactan sobre los objetivos del proyecto, bajo un esquema de rentabilidad, aplicando un mecanismo de control que compare los riesgos actuales con los datos históricos de proyectos pasados de la organización; para ello se utilizan como herramienta de análisis las redes neuronales artificiales, siguiendo el modelo de validación de eficiencia adoptado por Sarcià, Cantone y Victor R. BasiliBasili (2007). En este artículo se proporciona una visión general de los elementos del riesgo; asimismo, se revisan las características comunes de las redes neuronales artificiales, y se analiza la viabilidad de su aplicación en las organizaciones empresariales del sector construcción, que en los últimos años han tenido un crecimiento acelerado, mediante una aplicación práctica en un grupo de compañías de dicho sector.

El objetivo de este artículo es desarrollar un modelo, mediante el uso del análisis de redes neuronales artificiales, que permita determinar la probabilidad de éxito o fracaso de un proyecto de infraestructura antes de su inicio, para que la gerencia de la empresa pueda definir las estrategias y los planes de monitoreo o mitigación de riesgos.

\section{Descripción del problema}

\subsection{Realidad problemática}

El sector construcción es uno de los sectores productivos que lidera el dinamismo económico en el Perú y se ha convertido en uno de sus grandes 
motores. En el año 2011, y a pesar de la crisis, dicho sector creció en un 6,9\%. La agencia calificadora de riesgo Standard \& Poor's proyecta que la economía peruana crecerá 5,5\% en el 2012, mientras que la inflación sería de un 2,5\%. El mencionado informe proyecta una sólida recuperación de América Latina para el 2012, y que el Perú será el segundo país con mejor crecimiento en la región. Sin embargo, el desarrollo de este sector no ha sido siempre así, sino que ha estado sujeto a constantes cambios y al riesgo del ciclo del sector inmobiliario.

Actualmente, en el Perú las empresas que han tenido un desarrollo acelerado son las constructoras, debido al dinamismo positivo del mercado; no obstante, en este sector en general no tiene una visión del riesgo empresarial y se caracterizan por no contar con un departamento de riesgos. Son contadas las empresas constructoras que han implementado un área destinada a la gestión del riesgo, pero corren el peligro de direccionar sus conclusiones y recomendaciones a las metas de sus directivos, con una visión cortoplacista de la evolución del mercado.

En conclusión, no existe una cultura de prevención del riesgo, y solo algunas compañías han tomado la decisión de gestionar el riesgo con una visión integrada de la relación entre la rentabilidad esperada y el riesgo asumido por la empresa, y generar un proceso inteligente para predecir la evolución del mercado, con la finalidad de ajustar su capacidad productiva y lograr un estado de predicción permanente, con la posibilidad de dar la alarma necesaria en el momento oportuno, dentro de un mercado que se comporta de forma dinámica y fluctuante.

Se necesitan nuevos modelos de gestión del riesgo integrado en la empresa, que tengan la capacidad de alertar acerca de los efectos de la crisis mundial, que si bien no han alcanzado del todo al Perú, se debe estar preparados y aprender de lo sucedido en la economía global. En nuestro país se ha visto, hace algunos años, la desaparición y liquidación de empresas constructoras emblemáticas como el Grupo Gremco, la Corporación Sagitario, Constructora Villasol, entre otras, y en el mundo global se produjo la quiebra de grandes, medianas y pequeñas empresas, arrastradas por su falta absoluta de previsión por tener un departamento de riesgos que no cumple su función o, como en el caso del Perú, por carecer de este.

Se puede afirmar, entonces, que hoy se ha hecho imprescindible contar con nuevos modelos aplicables a la gestión de riesgos y la previsión (Smith 1990) para la toma de decisiones empresariales en la búsqueda de lograr los objetivos propuestos por la compañía y su sostenimiento. De esta manera, tanto en el Perú como en cualquier parte del mundo, ya no será un lujo sino una necesidad establecer un modelo de gestión de riesgos integrado para una empresa mediana o pequeña. 


\subsection{Definición del problema}

En el Perú las compañías constructoras no consideran a la gestión del riesgo como una área importante dentro de su organización y pocas son las que tienen un departamento de riesgo. Por lo tanto, se hace necesario realizar una evaluación del riesgo que asume una empresa frente a la propuesta de iniciar un nuevo proyecto y tomar la decisión de aceptarlo o descartarlo.

El principal problema que enfrenta el departamento de riesgo de una empresa constructora cuando aplica el proceso general de gestión del riesgo durante la fase de análisis de riesgo es poder calcular, con el mínimo error posible, la probabilidad de éxito o fracaso de un proyecto de infraestructura antes de iniciarse (su exposición al riesgo), a fin de que la gerencia esté en condiciones de decidir si lo ejecuta o lo descarta, confirmando las predicciones de éxito o de fracaso del proyecto, o realizando un replanteo de estas. Sin embargo, puede darse el caso de que una empresa constructora que no tenga implementado un departamento de riesgo logre realizar un cálculo subjetivo de la probabilidad de éxito o fracaso de un proyecto antes de que este sea iniciado, basándose en consultas a expertos o a todas las partes interesadas en él, lo que debería brindar una calificación subjetiva para cada riesgo identificado. No obstante, este proceso de estimación del riesgo contiene demasiada incertidumbre y no puede ser calificado como confiable.

Asimismo, es posible que el departamento de riesgo de la empresa realice un cálculo objetivo y estime el riesgo del proyecto en base a una evaluación de variables mediante la comparación de valores umbrales con proyectos similares ejecutados, pronosticando su clasificación, antes de ser iniciado, como éxito o fracaso. Por otra parte, puede ocurrir que los valores umbrales considerados por el departamento de riesgo de una empresa sean inciertos, ya que se basan en una media ponderada de medidas de exposición al riesgo, obtenidos de los datos históricos de la organización; o que la empresa adopte valores teóricos de comparación (por ejemplo valores pertenecientes a otras organizaciones), para predecir el éxito o el fracaso de un proyecto. En ambos casos el proceso de estimación del riesgo contiene demasiada incertidumbre y no es confiable.

Para revertir la situación expuesta, a continuación se plantean las siguientes interrogantes:

¿Cómo lograr calcular con el mínimo error posible la probabilidad de éxito o de fracaso de un proyecto antes de ser iniciado?

¿Es posible volver inteligente el proceso general de gestión del riesgo en la fase de análisis de riesgo, de modo que pueda predecir de manera confiable la probabilidad de éxito o fracaso de un proyecto de infraestructura antes de ser iniciado? 
¿Es posible construir un modelo basado en técnicas de inteligencia artificial, que pueda calcular la probabilidad de éxito o fracaso de un proyecto de infraestructura antes de ser iniciado?

\section{Marco teórico}

\subsection{Proceso de gestión del riesgo}

La gestión del riesgo como herramienta estratégica para la toma de decisiones empresariales es un proceso que busca disminuir la incertidumbre y lograr un estado de predicción permanente; mantiene una relación sinérgica y recursiva con la toma de decisiones y con la gestión integral de todos los procesos de la compañía, procurando su sostenibilidad.

El propósito de la gestión del riesgo es proveer información para la toma de decisiones empresariales reduciendo la incertidumbre y los efectos negativos del riesgo. Es un proceso continuo cuyo objetivo es aplicar las herramientas adecuadas, los procedimientos y las metodologías para evitar que el riesgo se concrete o mantenerlo dentro de los límites establecidos. Diversos estudios describen los pasos básicos de la gestión del riesgo en formas ligeramente diferentes, pero sustancialmente reportan un proceso similar al estándar australiano/neozelandés (AS/NZ 4360:2004) creado por la junta de estándares de Australia y Nueva Zelanda en el año 1999. La figura 1 muestra los principales elementos del proceso general de administración del riesgo de acuerdo con el estándar mencionado.

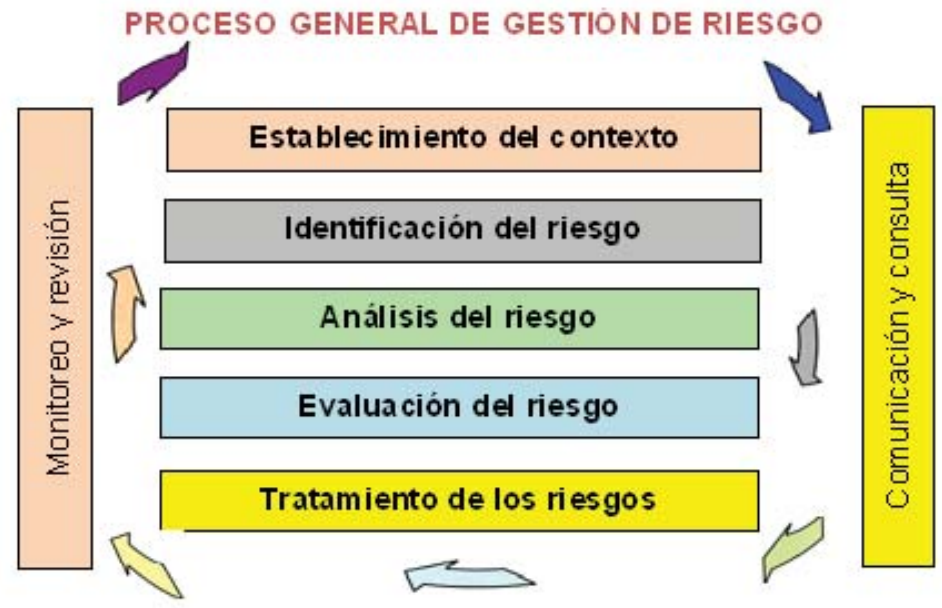

Figura 1. Proceso de gestión del riesgo

Fuente: Standards Australia (1999). 
A continuación se describen las etapas del proceso general de gestión del riesgo (Galarce, Pomier y Pastén 2006):

a) Establecimiento del contexto.- En esta etapa se establecen los contextos estratégico, organizacional y de administración de los riesgos. Se definen los criterios y parámetros básicos contra los cuales se evaluará el riesgo, así como su estructura de análisis.

b) Identificación del riesgo.- Se identifican las fuentes externas e internas del riesgo en relación con los objetivos de la organización.

c) Análisis del riesgo.- Se evalúa la probabilidad de ocurrencia del riesgo, considerando el rango de consecuencias potenciales, por medio de consultas directas a las partes interesadas a fin de estimar un nivel de riesgo.

d) Evaluación del riesgo.- Se compara el nivel de riesgo estimado contra el criterio preestablecido por la empresa (Galarce et al. 2006). La evaluación del riesgo es una actividad continua que vigila el estado del proyecto y comprueba los indicadores de proceso de gestión del riesgo. En esta etapa se recopilan los datos relativos a la tendencia del riesgo. Si el nivel alcanzado es alto se considera un riesgo explosivo, y si es bajo se considera un riesgo aceptable.

e) Tratamiento de los riesgos.- Los riesgos aceptables son monitoreados y no necesitan tratamiento. Sin embargo, se deben desarrollar e implementar las acciones de control para reducir los riesgos de tendencia explosiva.

f) Monitoreo y revisión.- Se monitorea y evalúan las correcciones que deben adoptarse en el plan de mitigación de riesgos. Un plan de contingencia se activará si los indicadores exceden al umbral fijo establecido.

g) Comunicación y consulta.- Se establece el intercambio de información mediante la comunicación y consulta apropiada con las partes interesadas (stakeholders) en la totalidad del proceso. Esta etapa especifica los componentes del proceso de administración de riesgos, pero no pretende establecer un parámetro invariable y uniforme en el sistema de administración del riesgo; por el contrario, es genérico, independiente y adaptable a cualquier sector industrial o económico (Galarce et al. 2006).

\subsection{Redes neuronales}

Una labor importante de la ciencia y la ingeniería ha sido el desarrollo de funciones que expliquen el comportamiento presente y pasado de un sistema, y las funciones que predigan su comportamiento; sin embargo, no todos los sistemas pueden ser modelados mediante funciones matemáticas que vaticinen su comportamiento futuro. Algunos ejemplos interesantes son pronosticar el comportamiento de la bolsa de valores, el de las variables macroeconómicas y financieras, y el riesgo 
empresarial. En los últimos años, las redes neuronales artificiales han emergido como una herramienta para el diseño de modelos de predicción. Los modelos de redes neuronales han demostrado su efectividad en la identificación de patrones intrincados, aprender de la experiencia, y predecir el comportamiento de sistemas con información ruidosa.

Los seres humanos somos redes creando redes, lo que se busca es crear una red artificial isomorfa a la red del sistema nervioso central, es decir, generar un modelo matemático que tenga la misma estructura de las redes neuronales del sistema nervioso, donde se reproduzcan algunas características importantes de su estructura natural, como son la distribución, redundancia y adaptabilidad. La característica de distribución se refiere al paralelismo que posee el cerebro, se estima un aproximado de cien mil millones de neuronas trabajando en paralelo, y que cada neurona tiene la capacidad de transmitir una señal de una célula a otra, por medio de un proceso conocido como sinapsis, con alrededor de unas 10.000 neuronas a la vez, y que toda la información almacenada en su vida está distribuida en toda la red. La característica de redundancia se refiere a la cualidad de tolerancia a fallos que tiene el cerebro, las neuronas son las únicas células del organismo que no se regeneran, y alrededor de los 25 años el ser humano empieza a perder neuronas que jamás son recuperadas, pero esta pérdida no merma en absoluto ninguna de las capacidades de nuestro sistema neuronal (Isasi y Galván 2004). La característica de adaptabilidad se refiere a la capacidad de aprendizaje que tiene el ser humano; el cual es un ser hermenéutico, es decir, aprende por medio de símbolos y ejemplos.

Cada neurona tiene entradas llamadas dendritas que condicionan el estado de su única salida, el axón, que está conectada a una dendrita de otra neurona mediante el proceso de la sinapsis, conforme se aprecia en la figura 2. El cuerpo de la neurona o soma contiene el núcleo, el mismo que se encarga de todas las actividades metabólicas de la neurona y recibe la información de otras neuronas vecinas a través de las conexiones sinápticas. Las dendritas envían los impulsos a otras células nerviosas, mediante una unión que puede ser de inhibición o de excitación según el neurotransmisor que se libere.

La sinapsis es un proceso químico donde se liberan substancias transmisoras, neurotransmisores, del lado del emisor de la unión, que eleva o disminuye el potencial eléctrico dentro del cuerpo de la célula receptora. Si el potencial sobrepasa el umbral se envía un pulso de información por el axón. Cuando se realiza cualquier actividad de aprendizaje, se entrenan las neuronas mediante el éxito o fracaso de una acción y las conexiones sinápticas del sistema nervioso se fortalecen, formándose enlaces y conexiones conocidas entre grupos de neuronas, que pueden servir para realizar otras acciones más complejas. 


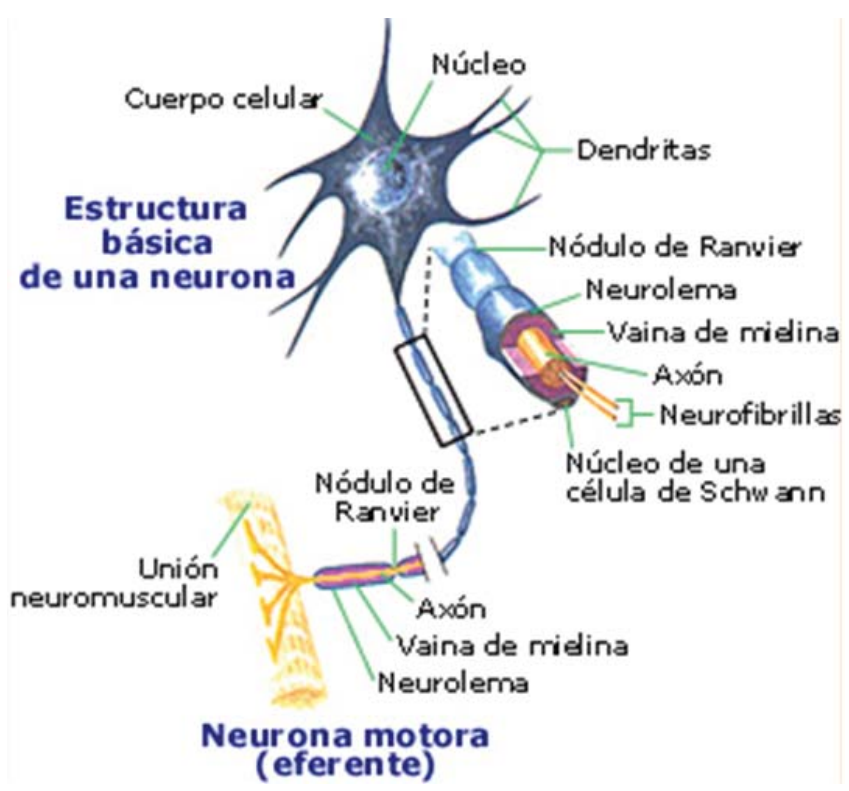

Figura 2. La estructura física de una neurona biológica

Fuente: Berardinis (1992).

\subsubsection{Redes neuronales artificiales}

Las redes neuronales artificiales forman parte de un conjunto de metodologías emergentes que se usan como soporte de trabajo de las organizaciones inteligentesy que actualmente tienen la denominación de análisis avanzado. Las redes neuronales artificiales no son programadas, aprenden a partir de ejemplos; normalmente a una red neuronal artificial se le presenta una serie de patrones, ejemplos, a través de los cuales ellas deben aprender. Esto supone un enfoque radicalmente distinto a los clásicos sistemas de software; en los programas de cómputo cada paso que el computador ejecuta debe ser anteriormente especificado por el programador, un solo proceso absorbe tiempo y recursos. Las redes neuronales comienzan con ejemplos de entradas y salidas y aprenden a producir la salida correcta para cada entrada. El enfoque de las redes neuronales consiste en que no requieren identificación de características, ni el desarrollo de algoritmos y programas para la resolución de problemas particulares. Sin embargo, presentan dos desventajas: el tiempo de aprendizaje de la red no se conoce a priori, y el diseño de una red por prueba y error puede ser muy complejo. Esto quiere decir que mientras la red no haya concluido su proceso de aprendizaje no debe ser descartada (Isasi y Galván, 2004). 
Las redes neuronales artificiales codifican la información de manera distribuida; es almacenada en la red y compartida por todas la unidades del proceso. Dicho esquema está en evidente contradicción con los esquemas tradicionales, donde los datos se encuentran almacenados en posiciones fijas de memoria. La información es almacenada en forma redundante y el resultado es un sistema tolerante a los fallos, lo que está en concordancia con la tolerancia a los fallos de nuestros propios sistemas neuronales.

Actualmente existen varios modelos de redes neuronales artificiales que son usados en diversos campos; destacan los modelos compilados en Michie, Spiegelhalter y Taylor, el cual es considerado como el estudio comparativo más completo entre redes neuronales artificiales y modelos estadísticos orientados a la clasificación (Sarle 2002), el modelo de la red de propagación hacia atrás (Rumelhart, Hinton y Williams 1986) y los mapas autoorganizados de Kohonen (1982). Estas estructuras pueden ser utilizadas en la clasificación y predicción del comportamiento de sistemas no lineales con información borrosa o incompleta, como es el caso de la gestión del riesgo. Un esquema de la red de propagación hacia atrás se muestra en la figura 3.

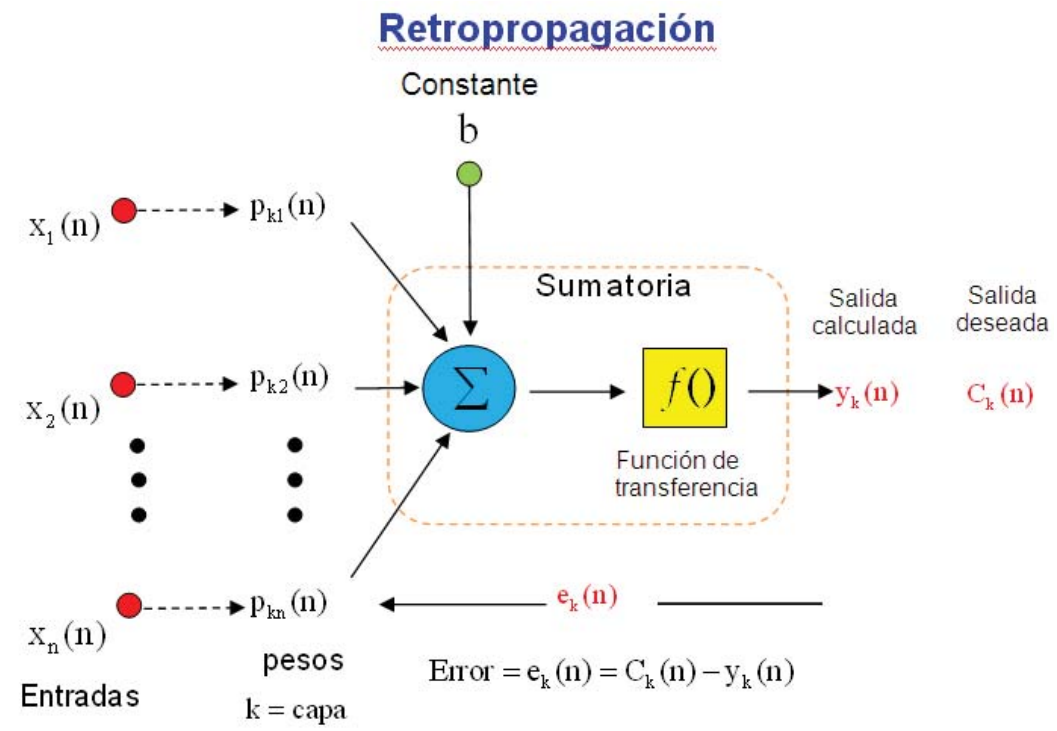

Figura 3. Estructura de la red neuronal en retropropagación

Fuente: Rumelhart et al. (1986). 
Una propuesta confiable para reducir la incertidumbre del cálculo del riesgo objetivo de un proyecto antes de ser iniciado, consiste en establecer un modelo que utilice los datos históricos de proyectos similares ejecutados por la empresa, con el fin de identificar los factores de riesgo que afecten las variables de éxito (Sarcià et al. 2007) del proyecto. El modelo propuesto usa como herramienta de análisis las redes neuronales artificiales, el cual tendrá como salida, variable de evaluación estimada, el indicador del objetivo del proyecto (efectividad, eficiencia y calidad) y el cálculo del riesgo en términos de la probabilidad de éxito o fracaso del proyecto antes de su ejecución. Esta medida estimada del riesgo debe definir las estrategias y planes de monitoreo o mitigación de riesgos.

Durante la etapa de evaluación del riesgo surge el problema de averiguar en qué medida los riesgos pueden repercutir en los objetivos del proyecto. Por ejemplo, si los riesgos impactan demasiado sobre el proyecto se obtiene baja probabilidad de éxito. Sin embargo, hay que tener en cuenta la base o umbral de comparación que arroja el modelo. Por ejemplo, supóngase a la efectividad como objetivo del proyecto, la cual se mide por el índice de efectividad (resultados alcanzados sobre resultados planificados), a fin de definir el éxito o el fracaso de un proyecto, el cual se compara con los índices de efectividad de proyectos similares de la empresa. Por ejemplo, supóngase que un proyecto obtiene un índice de efectividad E1 entre 0,7 y 0,8 . Si se define un índice de efectividad teórico de éxito $E 2=1,0$ como umbral de comparación, entonces, el proyecto es considerado como fracaso, ya que E1 < E2. Por el contrario, si se eligió como umbral el valor que arroja el modelo basado en redes neuronales artificiales, teniendo como dato la información de todos los proyectos similares ejecutados por la organización (y esta fuera, E3 $=0,65$ ) se tendría éxito (E1 > E3). Esto último ocurre porque el objeto de comparación se basa en experiencias reales de la organización. Por lo tanto, se procurará determinar el criterio de evaluación de los datos observados en base a las actuaciones reales de la empresa, mediante información relevante y actualizada, con el fin de formular un modelo adecuado que use la herramienta de las redes neuronales artificiales.

La estrategia que se propone está basada en el diseño de indicadores históricos comunes a los proyectos de una organización, que permitan construir una base de datos históricos de su comportamiento en la ejecución de proyectos similares, con el fin de calcular el impacto de los factores de riesgo sobre los objetivos del proyecto. El propósito de esta estrategia es generar una base de comparación del valor del riesgo. Por otra parte, se debe tener especial cuidado al definir los factores de riesgo, procurando identificar factores característicos del negocio, de modo que estos no resulten teóricos, irreales o de medición complicada. Se debe lograr que los datos tengan la mayor exactitud posible y puedan ser obtenidos con facilidad. Además, deben identificarse los factores del riesgo que tienen impacto positivo 
o negativo en los objetivos de un proyecto de infraestructura. A fin de evaluar el valor del riesgo, debe medirse la variación del impacto de los factores del riesgo en los objetivos.

Este documento propone definir los objetivos de un proyecto bajo el esquema de rentabilidad descrito en la figura 4. El esquema permite identificar los indicadores del negocio, relacionando elementos cuantificables para luego transformarlos a indicadores básicos. El esquema de rentabilidad está sostenido por tres indicadores generales: efectividad, eficiencia y calidad (Cruz 2007).

Con el fin de comprobar si un proyecto está cumpliendo con los objetivos expuestos, se consideran los umbrales de efectividad, eficiencia y calidad; de esta manera, el éxito o el fracaso del proyecto se definen según cómo se establecen estos umbrales. Esta interpretación permite definir un proyecto como exitoso, sobre la base de un aspecto específico (por ejemplo, un proyecto puede considerarse exitoso si el factor de efectividad supera o iguala el umbral establecido por el modelo, en caso contrario el proyecto se considera como un fracaso). Este concepto puede ser representado por una función de muchas variables, que calcula la métrica elegida para representar el éxito.

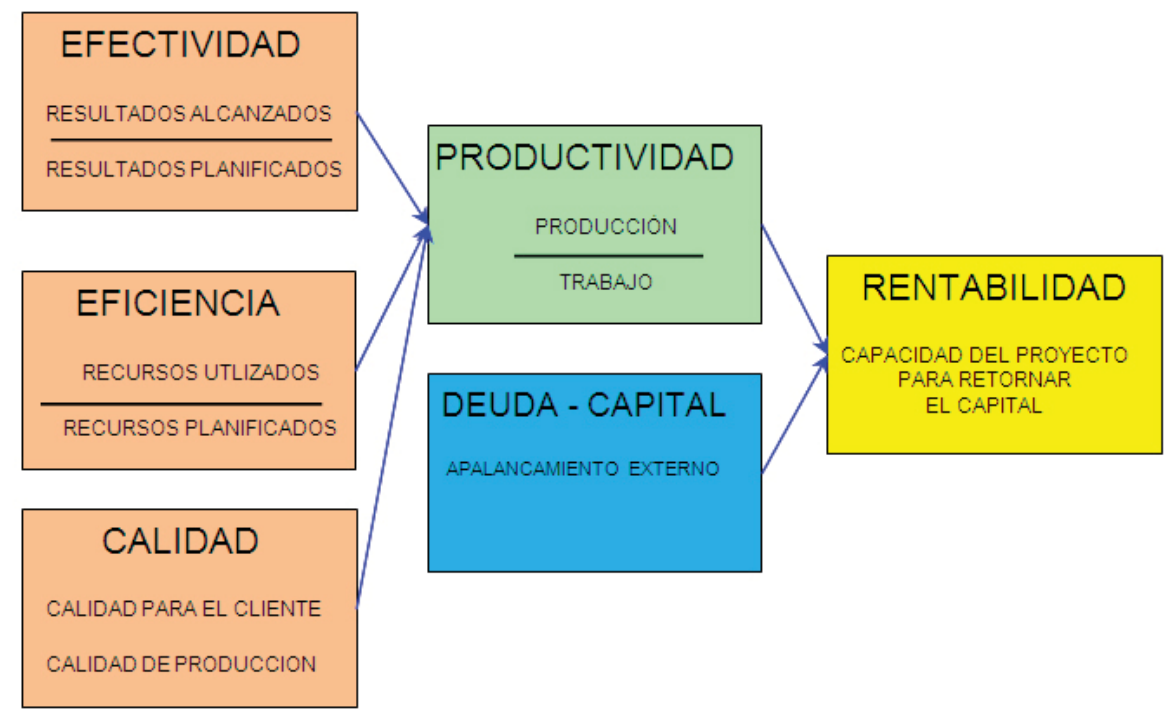

Figura 4. Esquema de rentabilidad

Elaboración propia. 
La figura 5 proporciona un esquema asociado a la presente propuesta. Asimismo, al usar la herramienta de redes neuronales artificiales puede calcularse la probabilidad de éxito para el proyecto actual; si esta probabilidad es inferior al umbral escogido, el impacto de los factores de riesgo aumenta, y tiene que activarse un plan de contingencia para mitigar el riesgo; caso contrario, si esta probabilidad es superior al umbral definido, el impacto de los factores de riesgo disminuye, por lo tanto la influencia de los factores de riesgo sobre los objetivos del proyecto (esquema de rentabilidad) es mínima y manejable en la etapa de monitoreo del proceso de gestión del riesgo.

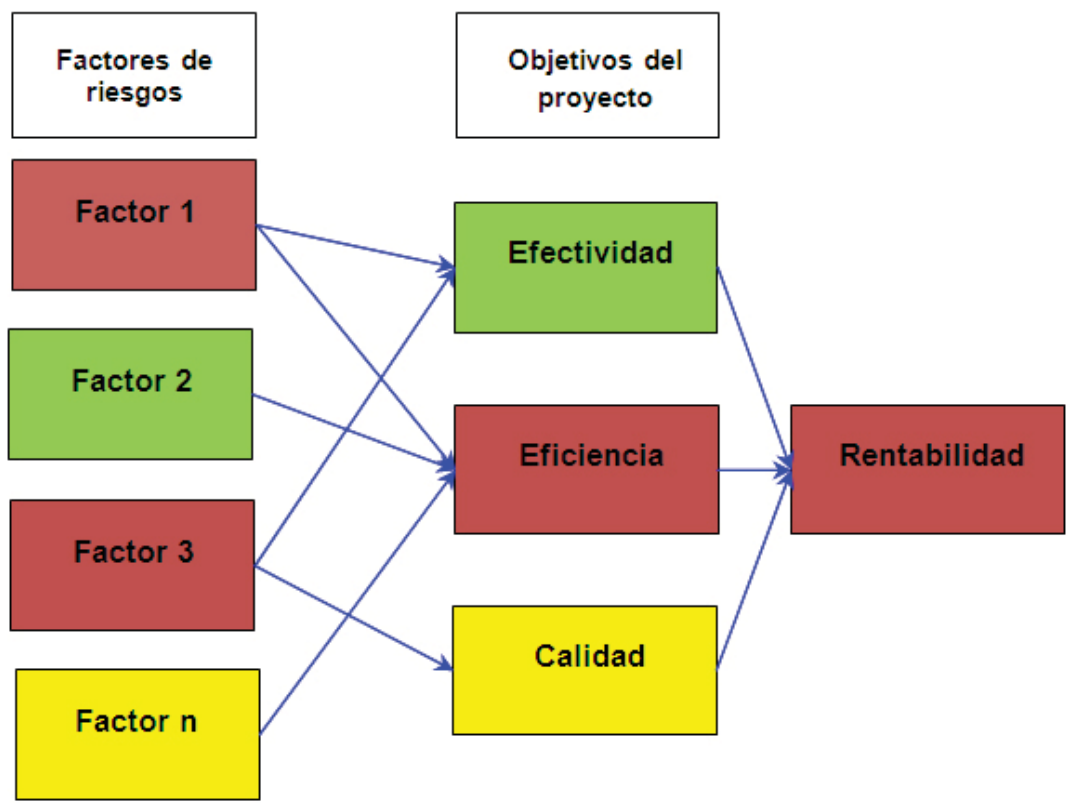

Figura 5. Impacto de los factores del riesgo en el esquema de rentabilidad

Elaboración propia.

Los datos históricos basados en los factores del riesgo permitirán construir una función de regresión para la evaluación del riesgo, ya que posibilita generar como salida los umbrales de comparación de los objetivos del proyecto. Esta función es generada al usar como herramienta las redes neuronales artificiales, cuyo resultado representa la métrica elegida para representar el éxito. 


\section{Metodología de solución}

La figura 6 describe el impacto de los factores de riesgo sobre los objetivos del proyecto con base en los datos históricos de la organización. Sin embargo, tienen que definirse los indicadores adecuados que permitan medir si los objetivos de un proyecto (efectividad, eficiencia y calidad) son alcanzados mediante la comparación de umbrales, a los que se denominarán indicadores de objetivo. Asimismo, se deben determinar los indicadores que definen los factores de riesgo y que impactan sobre los objetivos del proyecto, a los que se denominarán indicadores de factores de riesgo.

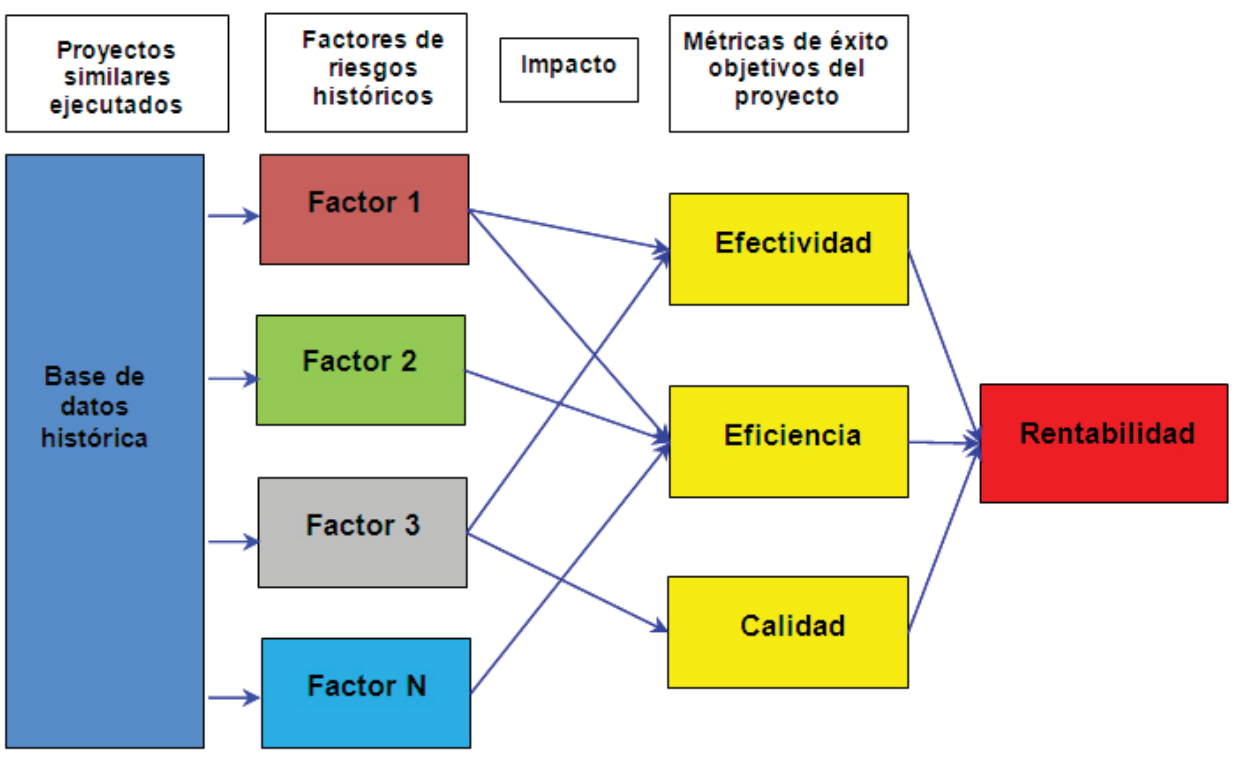

Figura 6. Base de datos histórica de proyectos similares ejecutados

Elaboración propia.

\subsection{Definición de indicadores}

\subsubsection{Indicadores de objetivos}

A continuación se definen los indicadores de objetivos que son medidas de éxito o fracaso en relación con los objetivos de un proyecto de infraestructura, a los cuales se denominarán métricas de éxito del proyecto. Los indicadores son definidos para el caso de una empresa constructora de obras de infraestructura tomando como referencia el modelo de gestión de indicadores de Salgueiro (2001). 
- Indicador de efectividad.- Este indicador proporciona información cuantificable sobre el cumplimiento del objetivo planteado (utilidad), y representa la capacidad del proyecto para alcanzar la utilidad programada. En el siguiente cuadro se diseña el indicador de efectividad propuesto.

\begin{tabular}{|l|c|}
\hline \multicolumn{1}{|c|}{ Descripción del indicador } & Medición \\
\hline $\begin{array}{l}\text { Efectividad en las utilidades del proyec- } \\
\text { to }(\mathrm{EFU}) \text {. Se define como el grado de } \\
\text { cumplimiento del plan de presupues- } \\
\text { to económico del proyecto ejecutado, } \\
\text { en términos de utilidad, y se denotará } \\
\text { como EFU. }\end{array}$ & EFU $=\frac{\text { Utilidad obtenida }}{\text { Utilidad programada }}$ \\
\hline
\end{tabular}

Tabla 1. Descripción del indicador de efectividad

Elaboración propia.

- Indicadores de eficiencia.- Representan la capacidad de lograr concluir el proyecto en el mínimo tiempo y con el menor costo unitario posible (Cruz 2007). En este sentido se propone el siguiente indicador de eficiencia:

\begin{tabular}{|l|c|}
\hline \multicolumn{1}{|c|}{ Descripción del indicador } & Medición \\
\hline $\begin{array}{l}\text { Relación del plazo de ejecución del } \\
\text { proyecto (RPEP). Indica la capacidad } \\
\text { de concluir el proyecto en el mínimo } \\
\text { tiempo posible. }\end{array}$ & $R P E P=\frac{\text { Plazo programado ejecución }}{\text { Plazo real ejecución }}$ \\
\hline $\begin{array}{l}\text { Nivel de inventarios (NI). Indica la efi- } \\
\text { ciencia del capital invertido en inventa- } \\
\text { rios respecto a las ventas netas del pro- } \\
\text { yecto. Eficiencia en el uso de insumos } \\
\text { del proyecto. }\end{array}$ & $\mathrm{NI}=\frac{\text { Costo del inventario }}{\text { ventas netas }}$ \\
\hline
\end{tabular}

Tabla 2. Descripción de los indicadores de eficiencia

Elaboración propia.

- Indicadores de calidad.- Representan la capacidad del proyecto para satisfacer al cliente, cuantifica la mejora continua de los procesos y resultados. Los indicadores de calidad propuestos son: 


\begin{tabular}{|l|c|}
\hline \multicolumn{1}{|c|}{ Descripción del indicador } & Medición \\
\hline $\begin{array}{l}\text { Rendimiento de calidad (RC). Mide la ca- } \\
\text { lidad del proceso, permite detectar las defi- } \\
\text { ciencias en el proyecto (Apaza 2003). Es una } \\
\text { relación entre el avance de ejecución de obra } \\
\text { aprobado por el cliente o la supervisión del } \\
\text { proyecto (metrado ejecutado conforme) y el } \\
\text { avance de ejecución de obra realmente eje- } \\
\text { cutado (metrado ejecutado real). }\end{array}$ & $R C=\frac{\text { Metrado ejecución conforme }}{\text { Metrado ejecución real }}$ \\
\hline $\begin{array}{l}\text { Calidad del servicio (CS). Mide la calidad } \\
\text { del servicio del proyecto con base en la acep- } \\
\text { tación del cliente (Apaza 2003). Es una rela- } \\
\text { ción entre el avance de obra observado por } \\
\text { el cliente (metrado ejecutado observado) y el } \\
\text { avance de ejecución de obra total (metrado } \\
\text { ejecutado real). }\end{array}$ & CS $=\frac{\text { Metrado ejecución observado }}{\text { Metrado ejecución real }}$ \\
\hline
\end{tabular}

Tabla 3. Descripción de los indicadores de calidad

Elaboración propia.

- Indicadores de productividad.- Representan la capacidad de producción del proyecto, se mide al relacionar la variable producción con la variable trabajo. El indicador de productividad propuesto es:

\begin{tabular}{|l|c|}
\hline \multicolumn{1}{|c|}{ Descripción del indicador } & Medición \\
\hline $\begin{array}{l}\text { Productividad de la mano de obra (PMO). } \\
\text { Mide la contribución de la mano de obra al } \\
\text { volumen de producción del proyecto, medi- } \\
\text { do en toneladas por horas hombre trabaja- } \\
\text { das (Apaza 2003). }\end{array}$ & PMO $=\frac{\text { Volumen producción conforme }}{\text { Horas hombre trabajadas }}$ \\
\hline $\begin{array}{l}\text { Costo unitario de producción (CUP). Es un } \\
\text { indicador integral de productividad del pro- } \\
\text { yecto. Consiste en una relación entre la valo- } \\
\text { rización del metrado ejecutado y la cantidad } \\
\text { del metrado ejecutado declarado conforme } \\
\text { por el cliente (supervisión). }\end{array}$ & CUP $=\frac{\text { Valorización metrado ejecutado }}{\text { Metrado ejecutado conforme }}$ \\
\hline
\end{tabular}

Tabla 4. Descripción de los indicadores de productividad

Elaboración propia. 
- Indicador de apalancamiento.- Representa la capacidad de apalancamiento del proyecto, se mide relacionando la deuda y el patrimonio de la empresa. El indicador propuesto es:

\begin{tabular}{|l|c|}
\hline \multicolumn{1}{|c|}{ Descripción del indicador } & \multicolumn{1}{c|}{ Medición } \\
\hline $\begin{array}{l}\text { Relación deuda-capital (RDC). Mide el } \\
\text { nivel de apalancamiento del proyecto, con } \\
\text { recursos externos (Cruz 2007). }\end{array}$ & RDC $=\frac{\text { Deuda total }}{\text { Patrimonio }}$ \\
\hline
\end{tabular}

Tabla 5. Descripción del indicador de apalancamiento

Elaboración propia.

- Indicadores de rentabilidad.- Miden la efectividad de la administración del proyecto para controlar los costos y gastos, representa la capacidad del proyecto de retornar la inversión. Los indicadores propuestos son:

\begin{tabular}{|l|c|}
\hline \multicolumn{1}{|c|}{ Descripción del indicador } & Medición \\
\hline $\begin{array}{l}\text { Rentabilidad total (RT). Es la utilidad medi- } \\
\text { da en términos de la capacidad de los activos } \\
\text { disponibles del proyecto (Cruz 2007). }\end{array}$ & RT $=\frac{\text { Utilidad neta }}{\text { Activo total promedio }}$ \\
\hline $\begin{array}{l}\text { Margen neto (MN). Mide la rentabilidad en } \\
\text { función de las ventas del proyecto. }\end{array}$ & MN $=\frac{\text { Utilidad neta }}{\text { Ventas netas }}$ \\
\hline
\end{tabular}

Tabla 6. Descripción de los indicadores de rentabilidad

Elaboración propia.

\subsubsection{Indicadores de los factores de riesgo}

Durante la etapa de identificación de riesgos, de un proceso de gestión del riesgo, deben identificarse los eventos que podrían impedir, degradar, demorar o mejorar el logro de los objetivos de la empresa (por ejemplo, la utilidad esperada del proyecto). Estos eventos constituyen las fuentes externas o internas del riesgo en relación con los objetivos de la empresa, que se denominarán factores del riesgo.

Se debe tener especial cuidado al identificar los factores de riesgo, procurando identificar factores característicos del negocio, de modo que estos no resulten teóricos, irreales o de medición complicada. Se debe lograr que los datos sean 
lo más exactos posible y obtenidos con facilidad. Además, deben identificarse los factores del riesgo que tienen impacto positivo o negativo en los objetivos de un proyecto de infraestructura.

Los factores de riesgo que afectan o impactan las métricas de éxito del proyecto deben ser expresados por indicadores, que se calcularán para todo proyecto de infraestructura. Estos indicadores son las entradas al modelo propuesto de la figura 7, que tiene como salida la métrica de éxito del proyecto y que será expresada en una función de regresión no lineal. Para obtener buenos resultados el modelo requiere un gran número de indicadores de factores del riesgo; sin embargo, si el número de indicadores crece, la métrica de éxito del proyecto será más difícil de estimar utilizando la estadística clásica. Una solución a este problema es usar la herramienta de redes neuronales artificiales, en particular el modelo multicapa feed-forward Backpropagation para calcular los valores de las funciones de regresión, de modo que el número de factores considerados no influyen sobre el procedimiento (Sarcià et al. 2007). Este documento toma como referencia el modelo de validación de eficiencia adoptado por Sarcià et al. (2007), basado en el análisis de redes neuronales artificiales como un medio para estimar una función de regresión no lineal, incluso si el número de factores de riesgo es elevado.

Para identificar los factores de riesgo que afectan los objetivos del proyecto, se ha tomado como referencia, entre otros, el modelo Goal Question Metric (Basili, Caldiera y Rombach 1994). Si bien este modelo fue creado para estimaciones en proyectos de software, el modelo proporciona un método eficaz para identificar factores e indicadores, tanto del proceso como de los resultados de proyectos de infraestructura, considerando que un programa de medición puede ser más satisfactorio si es diseñado teniendo en cuenta los objetivos perseguidos. En este enfoque las preguntas potencialmente medibles ayudan a obsevar si se está alcanzando en forma exitosa la meta trazada. Algunas preguntas posibles son las siguientes: ¿qué factores repercuten en el logro del objetivo de proyecto?, ¿cuál es el contexto del proyecto?, ¿cuál es el contexto de la organización?

La siguiente tabla contiene la propuesta de los factores de riesgo para proyectos de infraestructura y definición de indicadores del riesgo para medir el impacto sobre los objetivos (métricas de éxito) del proyecto. La escala de medición se elaboró con base en los datos provenientes de las experiencias de proyectos similares ejecutados (valores máximos y mínimos) por empresas ejecutoras de obras y de los valores límites permitidos por la legislación vigente. Por ejemplo, el precio ofertado por la ejecución de obras públicas por una empresa constructora no puede ser menor al 0,9 ni mayor al 1,1 del presupuesto base establecido por la entidad estatal que requiere el servicio, de conformidad con el tercer párrafo del artículo 39 del Reglamento de la Ley de Contrataciones del Estado Peruano promulgado por el Decreto Supremo No 184-2008-EF. 


\begin{tabular}{|c|c|c|c|c|}
\hline $\begin{array}{l}\text { Factores } \\
\text { de riesgo }\end{array}$ & Indicador & Medición & Símbolo & $\begin{array}{c}\text { Escala } \\
\text { de medición }\end{array}$ \\
\hline \multirow{2}{*}{$\begin{array}{l}\text { Precio ofer- } \\
\text { tado del } \\
\text { proyecto }\end{array}$} & \multirow{2}{*}{$\begin{array}{l}\text { Relación presupuesto } \\
\text { ofertado y presupuesto } \\
\text { base }\end{array}$} & Presupuesto ofertado & \multirow{2}{*}{ RPP } & \multirow{2}{*}[0,9;1,10]{} \\
\hline & & Presupuesto base & & \\
\hline \multirow{2}{*}{$\begin{array}{l}\text { Gastos ge- } \\
\text { nerales del } \\
\text { proyecto }\end{array}$} & \multirow{2}{*}{$\begin{array}{l}\text { Relación de gastos ge- } \\
\text { nerales totales (fijos más } \\
\text { variables) y el costo di- } \\
\text { recto de la obra (presu- } \\
\text { puesto contratado me- } \\
\text { nos impuestos, gastos } \\
\text { generales y utilidades) }\end{array}$} & Gasto directo & \multirow{2}{*}{ RGP } & \multirow{2}{*}[0,0;0,5]{} \\
\hline & & Costo directo proyecto & & \\
\hline \multirow{2}{*}{$\begin{array}{l}\text { Utilidad } \\
\text { prevista del } \\
\text { proyecto }\end{array}$} & \multirow{2}{*}{$\begin{array}{l}\text { Relación utilidad y el } \\
\text { costo directo de la obra } \\
\text { (presupuesto contrata- } \\
\text { do menos impuestos, } \\
\text { gastos generales y utili- } \\
\text { dades) }\end{array}$} & Utilidad & \multirow{2}{*}{ RUP } & \multirow{2}{*}[0,0;1,0]{} \\
\hline & & Costo directo proyecto & & \\
\hline $\begin{array}{l}\text { Tipo de } \\
\text { proyecto }\end{array}$ & $\begin{array}{l}\text { 1. Edificación } \\
\text { 2. Saneamiento } \\
\text { 3. Energético } \\
\text { 4. Irrigación } \\
\text { 5. Carreteras y puentes }\end{array}$ & Tipo de proyecto & TP & {$[1,2,3,4,5]$} \\
\hline $\begin{array}{l}\text { Duración } \\
\text { del } \\
\text { proyecto }\end{array}$ & $\begin{array}{l}\text { Plazo de ejecución del } \\
\text { proyecto }\end{array}$ & Días & PE & {$[0 ; 1000]$} \\
\hline $\begin{array}{l}\text { Experiencia } \\
\text { en obras }\end{array}$ & $\begin{array}{l}\text { Experiencia del ingenie- } \\
\text { ro residente de obras }\end{array}$ & Años & EIR & {$[1 ; 40]$} \\
\hline $\begin{array}{l}\text { Experiencia } \\
\text { en seguri- } \\
\text { dad }\end{array}$ & $\begin{array}{l}\text { Experiencia del ingenie- } \\
\text { ro de seguridad }\end{array}$ & Años & EIS & {$[0 ; 40]$} \\
\hline $\begin{array}{l}\text { Experiencia } \\
\text { en Calidad }\end{array}$ & $\begin{array}{l}\text { Experiencia del ingenie- } \\
\text { ro de calidad }\end{array}$ & Años & EIC & {$[0 ; 40]$} \\
\hline $\begin{array}{l}\text { Experiencia } \\
\text { en adminis- } \\
\text { tración }\end{array}$ & $\begin{array}{l}\text { Experiencia de la geren- } \\
\text { cia de administración }\end{array}$ & Años & EGA & {$[1 ; 40]$} \\
\hline
\end{tabular}


(continuación)

\begin{tabular}{|c|c|c|c|c|}
\hline $\begin{array}{l}\text { Factores } \\
\text { de riesgo }\end{array}$ & Indicador & Medición & Símbolo & $\begin{array}{c}\text { Escala } \\
\text { de medición }\end{array}$ \\
\hline $\begin{array}{l}\text { Variación } \\
\text { de precios } \\
\text { de mate- } \\
\text { riales, equi- } \\
\text { pos, mano } \\
\text { de obra } \\
\text { del sector } \\
\text { construc- } \\
\text { ción por } \\
\text { inflación }\end{array}$ & $\begin{array}{l}\text { Factor de reajuste K. } \\
\text { Proviene de la fórmula } \\
\text { polinómica del proyec- } \\
\text { to, calculado con base } \\
\text { en los índices de precios } \\
\text { unificados del sector } \\
\text { construcción publicada } \\
\text { por el INEl. }\end{array}$ & $\begin{array}{c}\text { Factor de reajuste del } \\
\text { proyecto }\end{array}$ & K & {$[0,5 ; 2]$} \\
\hline
\end{tabular}

Tabla 7. Factores de riesgo y definición de indicadores

Elaboración propia.

\subsection{Modelo de predicción de la métrica de éxito}

El modelo de predicción tiene como finalidad la estimación de la métrica de éxito del proyecto representado por una función de regresión no lineal, utilizando para tal fin la herramienta de redes neuronales artificiales. La métrica de éxito elegida es el índice de efectividad en las utilidades del proyecto (EFU) y la estructura de la red neuronal artificial utilizada en el modelo es la de retropropagación conocida como red multicapa feed forward (Rumelhart et al. 1986), donde las variables de entrada son los indicadores de los factores de riesgo del proyecto, y la salida es la métrica de éxito del proyecto (estimación de función de regresión no lineal). Una descripción de este modelo, se aprecia en la figura 7, donde el modelo de predicción calcula la métrica de éxito del proyecto, lo que da como resultado el valor del indicador de efectividad EFU. En síntesis, el modelo permite predecir el indicador de efectividad EFU de un proyecto nuevo y, asimismo, sirve para generar una base de datos donde estén clasificados los proyectos ejecutados por la empresa como proyectos con éxito o fracaso. En particular, si para un proyecto su indicador de efectividad real es mayor que el indicador de efectividad estimado por el modelo de predicción (valor umbral estimado por la red neuronal) el proyecto se clasifica como éxito (1); en caso contrario se clasifica como un fracaso (0) y se almacena en la base de datos con los indicadores de los factores de riesgos que le corresponde. 


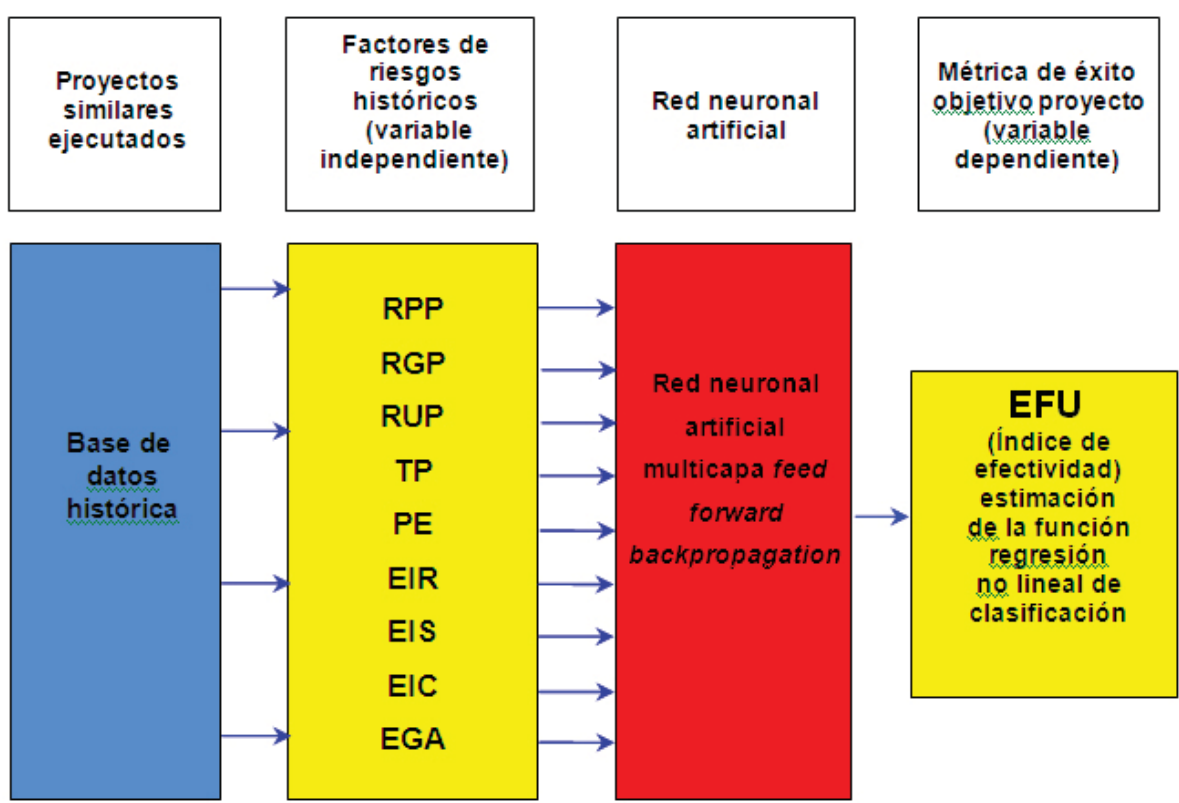

Figura 7. Modelo de predicción de la métrica de éxito

Elaboración propia.

\subsection{Clasificación de dos clases}

El modelo anterior permite clasificar los proyectos históricos de la empresa (proyectos ejecutados), en dos clases (éxito o fracaso), mediante la comparación del valor que arroja la red, que es el valor umbral del índice de efectividad en las utilidades (EFU), el cual es comparado con el EFU real del proyecto ejecutado, si el EFU real es mayor o igual que el EFU que arroja el modelo de predicción, entonces se clasifica como éxito, en caso contrario como fracaso. Considerando este esquema se puede construir una base de datos histórica donde los proyectos de infraestructura ejecutados por la empresa pertenecen a una clase éxito con clasificación 1 y a una clase fracaso con clasificación 0.

Asimismo, esta información permite estimar la probabilidad de éxito de un proyecto de infraestructura en general antes de que el proyecto se inicie. Efectivamente, a partir de los conjuntos de clasificación definidos por la red (clase proyecto éxito y clase proyecto fracaso) y basados en el modelo de validación de eficiencia (Sarcià et al. 2007) se puede calcular la probabilidad a priori del éxito proyecto de la siguiente forma:

$$
\begin{aligned}
& C_{E}=\text { Número de proyectos de la clase éxito } \\
& C_{F}=\text { Número de proyectos de la clase fracaso }
\end{aligned}
$$




$$
\text { Probabilidad de éxito de un Proyecto }=\frac{\mathrm{C}_{\mathrm{E}}}{\mathrm{C}_{\mathrm{E}}+\mathrm{C}_{\mathrm{F}}}
$$

\subsection{Modelo de predicción del éxito o fracaso}

Una vez clasificados los proyectos ejecutados por la empresa, como éxito (1) y fracaso (0), agrupados en forma conjunta con los indicadores de los factores de riesgo que determinan su clasificación, es posible aplicar un modelo que pueda predecir la probabilidad de éxito de un nuevo proyecto, indicando su tendencia de éxito o fracaso. Esta metodología implica el uso de una segunda red neuronal artificial feed forward donde las entradas son los indicadores de los factores de riesgo que afectan los objetivos del proyecto y la salida es un valor entre uno y cero que representa la probabilidad de éxito de un nuevo proyecto. Para esto se establece un patrón de aceptación o rechazo (fijado en 0,5 o más), lo cual permitirá aplicar las medidas de evaluación del modelo. En este caso, si el valor de salida es entre 0,5 y 1, se establece la tendencia de éxito del proyecto, y si el valor de salida es entre 0 y menos de 0,5 se establece la tendencia de fracaso del proyecto. Un esquema de este modelo se aprecia en la figura 8.
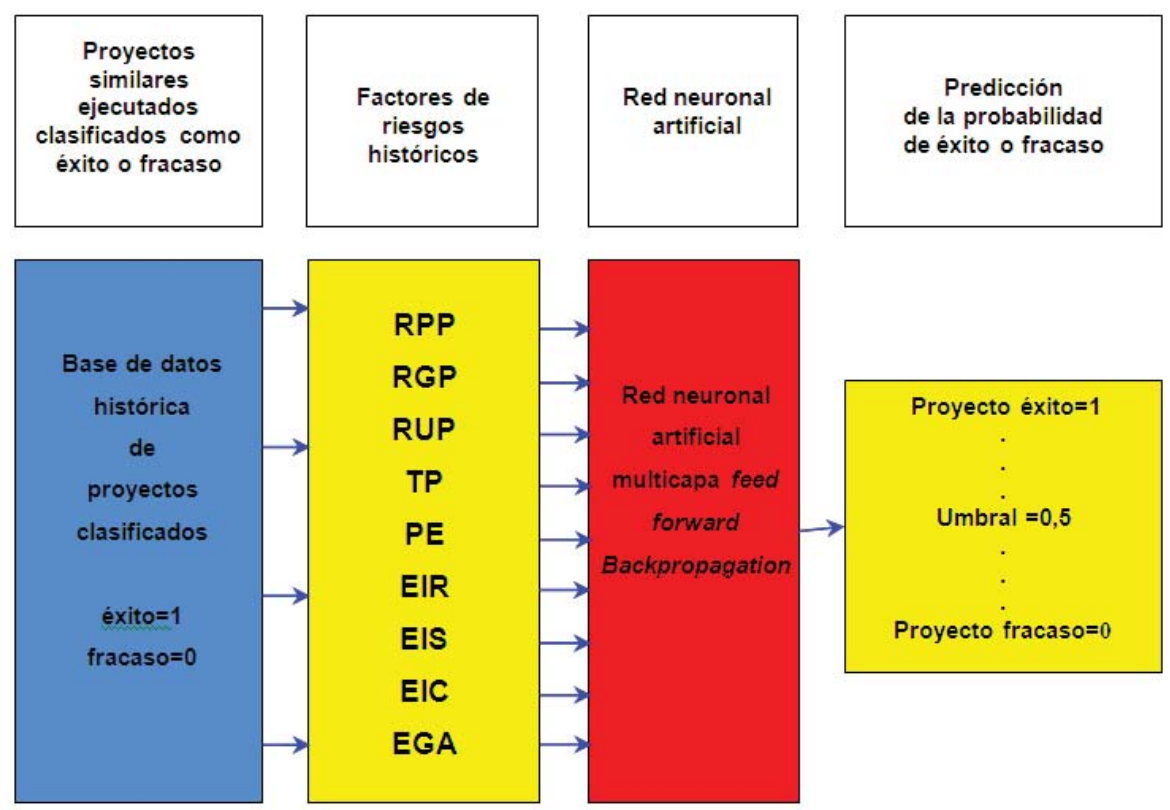

Figura 8. Predicción de la probabilidad de éxito o fracaso

Elaboración propia. 


\section{Caso de estudio}

En este caso se considera un conjunto real de observaciones constituido por la información de los proyectos ejecutados por Iconsa, un grupo constructor peruano fundado en 1986, formado actualmente por cinco empresas, el cual se dedica principalmente a la ejecución de obras de infraestructura para el Estado peruano. Se logró contar con la data histórica de 209 proyectos de construcción ejecutados por el grupo empresarial desde 1988. La información de cada proyecto se distribuye por empresa conforme a la siguiente tabla:

\begin{tabular}{|l|l|c|c|c|}
\hline Ítem & Empresas grupo Iconsa & $\begin{array}{c}\text { Número de } \\
\text { proyectos }\end{array}$ & $\begin{array}{c}\text { Datos por } \\
\text { proyecto }\end{array}$ & $\begin{array}{c}\text { Total } \\
\text { datos }\end{array}$ \\
\hline 1 & Iconsa & 70 & 11 & 770 \\
\hline 2 & Project Consulting S.A. & 69 & 11 & 759 \\
\hline 3 & Constructora San Gabriel S.A.C & 30 & 11 & 330 \\
\hline 4 & Constructora Amazonas S.A.C. & 10 & 11 & 110 \\
\hline 5 & Proice S.A.C. & 30 & 11 & 330 \\
\hline & & 209 & & 2299 \\
\hline
\end{tabular}

Tabla 8. Información de proyectos del grupo Iconsa

Elaboración propia.

En cada proyecto se identificaron diez indicadores; a continuación, en la tabla se resumen los indicadores usados para medir los factores de riesgo que afectan a los objetivos de cada proyecto:

\begin{tabular}{|c|l|l|c|c|c|c|}
\hline Ítem & $\begin{array}{l}\text { Factores } \\
\text { de riesgo }\end{array}$ & Indicador & Símbolo & $\begin{array}{l}\text { Escala de } \\
\text { medición }\end{array}$ & Paso & Cardinal \\
\hline 1 & $\begin{array}{l}\text { Precio ofertado } \\
\text { del proyecto }\end{array}$ & $\begin{array}{l}\text { Relación presu- } \\
\text { puesto contrata- } \\
\text { do y presupuesto } \\
\text { base }\end{array}$ & RPP & {$[0,90 ; 1,10]$} & 0,01 & 21 \\
\hline 2 & $\begin{array}{l}\text { Gastos genera- } \\
\text { les del proyecto }\end{array}$ & $\begin{array}{l}\text { Relación gastos } \\
\text { generales y el cos- } \\
\text { to directo del pre- } \\
\text { supuesto }\end{array}$ & RGP & {$[0,01 ; 0,50]$} & 0,01 & 50 \\
\hline 3 & $\begin{array}{l}\text { Utilidad prevista } \\
\text { del proyecto }\end{array}$ & $\begin{array}{l}\text { Relación utilidad } \\
\text { y el costo directo } \\
\text { del presupuesto }\end{array}$ & RUP & {$[0,01 ; 1,00]$} & 0,01 & 100 \\
\hline
\end{tabular}




\begin{tabular}{|c|l|l|c|c|c|c|}
\hline Ítem & $\begin{array}{l}\text { Factores } \\
\text { de riesgo }\end{array}$ & Indicador & Símbolo & $\begin{array}{l}\text { Escala de } \\
\text { medición }\end{array}$ & Paso & Cardinal \\
\hline 4 & $\begin{array}{l}\text { Tipo de proyec- } \\
\text { to }\end{array}$ & Tipo de proyecto & TP & {$[1,2,3,4,5]$} & 1 & 5 \\
\hline 5 & $\begin{array}{l}\text { Duración del } \\
\text { proyecto }\end{array}$ & $\begin{array}{l}\text { Plazo de ejecu- } \\
\text { ción del proyecto }\end{array}$ & PE & {$[1 ; 1000]$} & 20 & 50 \\
\hline 6 & $\begin{array}{l}\text { Experiencia en } \\
\text { obras }\end{array}$ & $\begin{array}{l}\text { Experiencia del in- } \\
\text { geniero residente }\end{array}$ & EIR & {$[1 ; 40]$} & 1 & 40 \\
\hline 7 & $\begin{array}{l}\text { Experiencia en } \\
\text { seguridad }\end{array}$ & $\begin{array}{l}\text { Experiencia del } \\
\text { ingeniero de se- } \\
\text { guridad }\end{array}$ & EIS & {$[0 ; 40]$} & 1 & 41 \\
\hline 9 & $\begin{array}{l}\text { Experiencia en } \\
\text { calidad }\end{array}$ & $\begin{array}{l}\text { Experiencia del } \\
\text { ingeniero de cali- } \\
\text { dad }\end{array}$ & EIC & {$[0 ; 40]$} & 1 & 41 \\
\hline 10 & $\begin{array}{l}\text { Experiencia en } \\
\text { administración }\end{array}$ & $\begin{array}{l}\text { Experiencia de la } \\
\text { gerencia de admi- } \\
\text { nistración }\end{array}$ & EGA & {$[1 ; 40]$} & 1 & 40 \\
\hline $\begin{array}{l}\text { Variación de } \\
\text { precios (mate- } \\
\text { riales, equipos, } \\
\text { mano de obra) } \\
\text { del sector cons- } \\
\text { trucción por in- } \\
\text { flación }\end{array}$ & $\begin{array}{l}\text { Factor de reajuste } \\
\text { K }\end{array}$ & K & {$[0,5 ; 2]$} & 0,1 & 16 \\
\hline
\end{tabular}

Tabla 9. Indicadores de los factores de riesgo que afectan a los objetivos de cada proyecto

Elaboración propia.

\subsection{Elección del modelo}

Las pruebas de clasificación se efectuaron con el modelo de red neuronal artificial multicapa feed forward (Rumelhart et al. 1986) usando el algoritmo de aprendizaje de retropropagación de Levenberg-Marquardt (gradiente descendente optimizado). A fin de mejorar la precisión de la red, se empleó una cantidad más amplia de datos, considerando un conjunto de prueba adicional al de validación. A diferencia del conjunto de validación, el conjunto de prueba no aporta ningún tipo de índice al entrenamiento de la red pues se trata de un indicador externo. El conjunto de 
validación aporta la razón de aprendizaje, que está en función al error cuadrático medio de la regresión con dicho conjunto. Se probaron 16 arquitecturas de redes neuronales artificiales. La distribución del número de capas como la cantidad de neuronas por capa se dio bajo la permutación de los números 5, 9, 10 y 20 (obtenidos por una función random de rango [2; 20]). El programa utilizado para la simulación y entrenamiento de la red fue el Neural Networks Toolbox (MatLab ${ }^{\mathrm{TM}}$ ); asimismo, se empleó el software Minitab Solutions para el cálculo de los datos estadísticos y la elaboración de histogramas. La función de transición empleada en la red perceptrón multicapa feed forward es la función sigmoidal para la capa de entrada y las capas ocultas, y una función lineal para la capa de salida de la red.

De los 209 conjuntos de datos empleados en la preparación de las redes neuronales se emplearon 171 en el conjunto de entrenamiento, 19 en el conjunto de validación y 19 en el de prueba. Para el entrenamiento se emplearon hasta dos métodos: máximo alcanzado (media) y gradiente mínimo alcanzado (razón de entrenamiento). Esto se debe a la necesidad de encontrar un mínimo local lo suficientemente bajo como para minimizar el error tanto en el conjunto de entrenamiento como en el de validación.

\subsection{Evaluación de la red}

El entrenamiento de las diferentes arquitecturas de redes neuronales artificiales se evaluó teniendo en consideración dos métricas: el grado de error y el rendimiento. Para la medición del grado del error de cada arquitectura se empleó el error porcentual absoluto medio (Mean Absolute Percent Error - MAPE) del conjunto de validación y del conjunto de prueba. Finalmente, la definición del mejor modelo se realizó en base al cálculo del error absoluto medio de ambos conjuntos.

Para la evaluación de los resultados de salida de las diferentes arquitecturas probadas se empleó el índice de rendimiento error cuadrático medio (Mean Square Error - MSE) relativo al conjunto de entrenamiento proporcionado por Matlab, y el error porcentual absoluto medio (MAPE) de los conjuntos de validación y prueba. Además, se calculó la desviación estándar del error absoluto (StDev) de ambos conjuntos para observar la estabilidad de la red. Los resultados se muestran en la tabla 9. 


\begin{tabular}{|c|c|c|c|c|c|c|c|}
\hline & \multirow{2}{*}{\begin{tabular}{|c|} 
Entrenamiento \\
MSE \\
\end{tabular}} & \multicolumn{2}{|c|}{ Total (\%) } & \multicolumn{2}{|c|}{ Validación (\%) } & \multicolumn{2}{|c|}{ Test (\%) } \\
\hline & & MAPE & StDev & MAPE & StDev & MAPE & StDev \\
\hline & 0,000888 & 5,96 & 5,25 & 3,91 & 4,71 & 7,51 & 5,09 \\
\hline $5 \times 9$ & 0,000177 & 9,78 & 12,25 & 3,61 & 3,55 & 15,95 & 14,71 \\
\hline $5 \times 10$ & 0,000543 & 9,34 & 6,38 & 6,28 & 5,18 & 11,98 & 6,34 \\
\hline $5 \times 20$ & 0,00573 & 16,76 & 12,97 & 12,07 & 10,87 & 21,33 & 13,77 \\
\hline $9 \times 5$ & 0,00687 & 8,05 & 4,84 & 5,38 & 4,22 & 10,19 & 4,03 \\
\hline $9 \times 9$ & 0,0000491 & 7,29 & 4,71 & 4,22 & 3,67 & 9,88 & 3,78 \\
\hline $9 \times 10$ & 0,00166 & 9,45 & 6,21 & 6,39 & 5,95 & 11,80 & 4,96 \\
\hline $9 \times 20$ & 0,00391 & 8,42 & 6,15 & 8,05 & 5,99 & 8,39 & 6,37 \\
\hline $10 \times 5$ & 0,0024 & 6,22 & 5,80 & 5,04 & 5,83 & 7,21 & 5,86 \\
\hline $10 \times 9$ & 0,000244 & 10,03 & 10,78 & 5,56 & 5,23 & 14,18 & 13,22 \\
\hline $10 \times 10$ & 0,00809 & 9,95 & 6,20 & 10,28 & 6,04 & 9,39 & 6,55 \\
\hline $10 \times 20$ & 0,000927 & 15,17 & 8,24 & 10,33 & 7,76 & 19,51 & 6,19 \\
\hline $20 \times 5$ & 0,000602 & 7,11 & 7,81 & 6,26 & 9,70 & 7,75 & 5,91 \\
\hline $20 \times 9$ & 0,000228 & 10,07 & 12,77 & 3,72 & 3,83 & 15,90 & 15,63 \\
\hline $20 \times 10$ & 0,000666 & 5,67 & 3,44 & 5,27 & 2,90 & 5,85 & 3,92 \\
\hline $20 \times 20$ & 0,00327 & 7,33 & 5,58 & 740 & 4,84 & 7,02 & 6,35 \\
\hline
\end{tabular}

Tabla 9. Errores medio y desviación estándar

Elaboración propia.

Los resultados mostrados en la tabla 9 indican que la arquitectura que presenta menor error y que mejor se ajusta para la predicción del modelo es la red feed forward 20 x 10 (20 capas ocultas, 10 neuronas en la capa escondida, 10 neuronas en la entrada y una neurona en la salida). Por lo tanto, se escogió la mencionada red como la más apta para la predicción del modelo debido al bajo porcentaje de error que obtiene en los conjuntos de validación y prueba. Se descarta el índice del rendimiento, pues es un índice referencial al entrenamiento y a la validación, además de haberse empleado métodos de detención del entrenamiento que impliquen una alta especialización en los datos de entrenamiento (lo cual hace que los índices del entrenamiento se vean sesgados). 


\subsection{Clasificación de los proyectos en dos clases: Éxito y fracaso}

Se procedió a clasificar los proyectos históricos de la empresa (proyectos ejecutados) en dos clases: éxito (1) o fracaso (0), mediante la comparación del valor que arroja la red: el valor umbral del índice de efectividad en las utilidades (EFU), el cual es comparado con el EFU real del proyecto ejecutado; si el EFU real es mayor o igual que el EFU que arroja el modelo de predicción, entonces el proyecto se clasifica como éxito, en caso contrario como fracaso. Considerando este esquema se puede construir una base de datos histórica donde los proyectos de infraestructura ejecutados por la empresa forman una clase éxito con clasificación 1 y una clase fracaso con clasificación 0.

Las salidas se determinaron según el signo de la diferencia entre el valor real y el valor estimado del índice EFU, en el caso de ser no nulo o positivo se califica el valor como 1 (éxito), en caso de ser negativo (si realmente se está por debajo del promedio) se califica como fracaso. Por ejemplo, si el valor estimado del índice EFU obtuvo un valor de 1,0 y el valor real del EFU es de 1,1 se puede inferir que el proyecto está por encima del promedio de la regresión, por lo cual es un hecho positivo (es una mejora). En caso contrario, si la red predice un valor del índice EFU de 1,1 y el valor real es 1,0; se puede decir que el proyecto no ha sido satisfactorio pues está por debajo del promedio histórico de la empresa (estimado por el modelo).

Cálculo de la probabilidad de éxito: El siguiente paso fue crear una matriz de datos con todas las combinaciones posibles de entrada. Esta etapa procesa la probabilidad de éxito de un proyecto de edificación $(T P=1)$ y restringe el valor de los índices. La tabla 10 muestra el detalle del número de elementos (cardinal) y rangos empleados por cada indicador utilizado, generando una matriz de datos con todas las combinaciones posibles de entrada ( $5 \times 5 \times 5 \times 3 \times 5 \times 2 \times 2 \times 2 \times 2 \times 5)$; el total de datos es 150.000 . 
Redes neuronales para la optimización de la gestión del riesgo empresarial

\begin{tabular}{|c|c|c|c|}
\cline { 2 - 4 } \multicolumn{1}{c|}{} & \multicolumn{2}{c|}{ Rango } & \multicolumn{2}{c|}{ Paso } & \multicolumn{2}{c|}{ Cardinal } \\
\hline EFU & \multicolumn{2}{c|}{ No se incluye en el cálculo } \\
\hline RPP & $0,9: 1,1$ & 0,05 & 5 \\
\hline RGP & $0,1: 0,5$ & 0,1 & 5 \\
\hline RUP & $0,2: 1$ & 0,2 & 3 \\
\hline TP & $01: 03$ & 1 & 5 \\
\hline PE & $200: 1000$ & 200 & 2 \\
\hline EIR & $01: 20$ & 19 & 2 \\
\hline EIS & $00: 20$ & 20 & 2 \\
\hline EIC & $00: 20$ & 20 & 2 \\
\hline EGA & $01: 20$ & 19 & 5 \\
\hline K & $0,98: 1,1$ & 0,3 & \\
\hline
\end{tabular}

Tabla 10. Tabla de índices restringidos para la prueba general

Elaboración propia.

Como se expresa en la tabla 10, se emplea la primera red, ya que se pretende hallar la probabilidad de éxito que tiene la red neuronal frente a la realidad (que viene a ser la probabilidad de que el proyecto sea mejor que el promedio histórico con el cual se inicializó la red o promedio histórico). Los resultados de los datos ingresados al modelo se resumen en el histograma mostrado en la figura 9, en el cual se puede observar la mayor predisposición al éxito que tienen los proyectos de edificación.

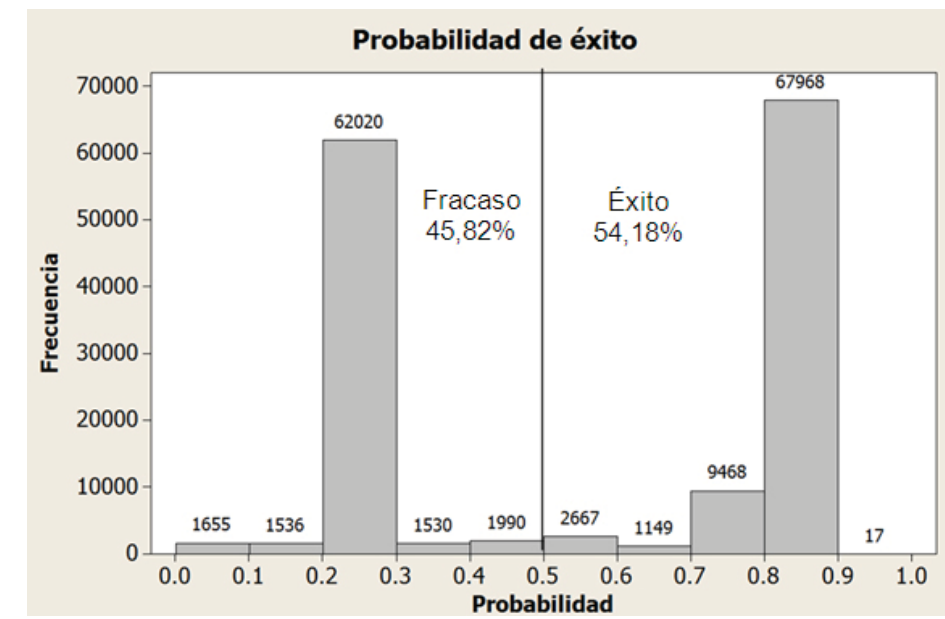

Figura 9. Histograma de las probabilidades de éxito

Elaboración propia. 
La línea divisoria de la figura 9 separa dos tendencias de los proyectos y el porcentaje que aparece bajo cada etiqueta $(45,82 \%$ y $54,18 \%)$ se refiere a la probabilidad de fracaso o de éxito que tiene cualquier proyecto de edificación antes de comenzar. Finalmente, se procedió a calcular la probabilidad a priori de éxito, basándose en los datos del histograma. La probabilidad a priori de éxito representa la probabilidad de ocurrencia de valores mayores a 0,5 en la red. Los resultados finales se aprecian en la tabla 11.

\begin{tabular}{|c|c|c|}
\hline Rango & Frecuencia & Porcentaje de incidencia \\
\hline $\mathbf{0 , 0 - 0 , 1}$ & 1655 & 1,10 \\
\hline $\mathbf{0 , 1 - 0 , 2}$ & 1536 & 1,02 \\
\hline $\mathbf{0 , 2 - 0 , 3}$ & 62020 & 41,35 \\
\hline $\mathbf{0 , 3 - 0 , 4}$ & 1530 & 1,02 \\
\hline $\mathbf{0 , 4 - 0 , 5}$ & 1990 & 1,33 \\
\hline $\mathbf{0 , 5 - 0 , 6}$ & 2667 & 1,78 \\
\hline $\mathbf{0 , 6 - 0 , 7}$ & 1149 & 0,77 \\
\hline $\mathbf{0 , 7 - 0 , 8}$ & 9468 & 6,31 \\
\hline $\mathbf{0 , 8 - 0 , 9}$ & 67968 & 45,31 \\
\hline $\mathbf{0 , 9 - 1 , 0}$ & 17 & 0,01 \\
\hline Total & $\mathbf{1 5 0 0 0 0}$ & $\mathbf{1 0 0 , 0 0}$ \\
\hline Fracaso & $\mathbf{6 8 7 3 1}$ & $\mathbf{4 5 , 8 2}$ \\
\hline Aceptación & $\mathbf{8 1 2 6 9}$ & $\mathbf{5 4 , 1 8}$ \\
\hline
\end{tabular}

Tabla 11. Tabla de resultados de probabilidades de fracaso y aceptación

Elaboración propia.

\subsection{Cálculo de la probabilidad de éxito o fracaso de un proyecto}

Una vez construida la base de datos que contiene todos los proyectos ejecutados por la empresa, con sus indicadores de los factores de riesgo y su respectiva clasificación como éxito (1) o fracaso (0), se procedió a implementar el modelo que prediga la probabilidad de éxito de un nuevo proyecto, indicando su tendencia de éxito o fracaso. Esta metodología implicó el uso de una segunda red neuronal artificial feed forward, donde las entradas son los indicadores de los factores de riesgo que afectan los objetivos del proyecto y la salida que se obtuvo es un valor entre cero y uno que representa la probabilidad de éxito de un nuevo proyecto. Por ejemplo, un valor de salida 1 indica que el proyecto será un éxito, y un valor 
cero que será un fracaso, pero si la salida es un valor fraccionario como 0,3 significa que existe una probabilidad de 0,3 de que el proyecto sea un éxito y de un 0,7 de probabilidad de que sea un fracaso.

Se optó por crear 16 arquitecturas de redes siguiendo los mismos patrones que en la primera red. Al igual que en la etapa anterior, se buscó analizar la performance de la red y el error porcentual absoluto medio (MAPE) para la elección de la mejor arquitectura y representación de los patrones. Los índices del aprendizaje se aprecian en la tabla 12.

\begin{tabular}{|c|c|c|c|c|c|c|c|}
\cline { 2 - 8 } \multicolumn{1}{c|}{} & Entrenamiento & \multicolumn{2}{c|}{ Total (\%) } & \multicolumn{2}{c|}{ Validación (\%) } & \multicolumn{2}{c|}{ Test (\%) } \\
\cline { 2 - 8 } \multicolumn{1}{c|}{} & MSE & MAPE & StDev & MAPE & StDev & MAPE & StDev \\
\hline $5 \times 5$ & 0,0702 & $\mathbf{3 0 , 3 6}$ & 31,20 & 17,37 & 25,54 & 42,42 & 32,59 \\
$5 \times 9$ & 0,0867 & $\mathbf{1 6 , 9 6}$ & 19,40 & 14,31 & 21,04 & 20,25 & 18,02 \\
$5 \times 10$ & 0,0132 & $\mathbf{2 1 , 0 6}$ & 41,16 & 13,04 & 26,99 & 29,65 & 51,31 \\
$5 \times 20$ & $\mathbf{0 , 1 8 6}$ & $\mathbf{4 5 , 7 4}$ & 20,35 & 34,84 & 20,17 & 55,08 & 15,39 \\
$9 \times 5$ & $\mathbf{0 , 0 7 2 3}$ & $\mathbf{2 3 , 1 6}$ & 34,45 & 11,70 & 23,76 & 34,58 & 40,39 \\
$9 \times 9$ & 0,209 & $\mathbf{4 5 , 7 8}$ & 25,39 & 34,05 & 24,88 & 56,19 & 21,87 \\
$9 \times 10$ & 0,19 & $\mathbf{4 7 , 1 2}$ & 17,71 & 36,91 & 18,82 & 56,25 & 10,59 \\
$9 \times 20$ & 0,189 & $\mathbf{5 2 , 8 6}$ & 35,54 & 37,91 & 36,42 & 64,13 & 28,60 \\
$10 \times 5$ & 0,0875 & $\mathbf{4 0 , 1 2}$ & 36,31 & 24,22 & 34,53 & 54,84 & 33,16 \\
$10 \times 9$ & 0,185 & $\mathbf{3 7 , 9 6}$ & 20,78 & 34,79 & 17,92 & 41,62 & 23,48 \\
\hline $10 \times 10$ & $\mathbf{0 , 0 6 0 6}$ & $\mathbf{1 1 , 7 6}$ & 23,68 & 10,29 & 23,45 & 13,62 & 24,96 \\
\hline $10 \times 20$ & $\mathbf{0 , 2 0 1}$ & $\mathbf{5 1 , 9 0}$ & 15,98 & 45,58 & 14,72 & 57,12 & 15,48 \\
$20 \times 5$ & 0,204 & $\mathbf{5 4 , 5 2}$ & 18,55 & 44,84 & 19,64 & 62,89 & 12,74 \\
$20 \times 9$ & 0,224 & $\mathbf{5 4 , 2 5}$ & 15,80 & 46,41 & 16,21 & 61,06 & 12,11 \\
$20 \times 10$ & 0,219 & $\mathbf{4 5 , 6 5}$ & 28,53 & 32,68 & 28,44 & 57,25 & 24,28 \\
$20 \times 20$ & 0,139 & $\mathbf{5 2 , 9 9}$ & 26,65 & 41,18 & 27,34 & 63,53 & 22,12 \\
\hline
\end{tabular}

Tabla 12. Errores medio y desviación estándar

Elaboración propia.

\subsection{Construcción de la interfaz de predicción de éxito}

Para culminar el caso de estudio se procedió a construir una interfaz visual que acepte los valores de los indicadores de los factores de riesgo del proyecto como datos de entrada, con el fin de predecir la métrica de éxito, que en el modelo es el indicador de efectividad (EFU) descrito en el ítem 4.1.1 A pesar de que se podría utilizar Matlab para la ejecución de la predicción, se descartó su uso por el grado 
de dificultad en el empleo del modelo escogido y el tiempo excesivo de ejecución que tarda en realizar toda la secuencia de simulación.

Se eligió construir una interfaz que se programó en el lenguaje JAVA, y se empleó el IDE Netbeans 7.0 Beta para su desarrollo. La ejecución de esta interfaz requirió JRE 6 (Java Runtime Environment) y un sistema operativo que soporte JRE6. La interfaz visual es un formulario, el cual tiene como parámetros los diez índices especificados para el empleo del modelo. También tiene opciones para cerrar la ventana, limpiar los campos y predecir el resultado según los datos ingresados.

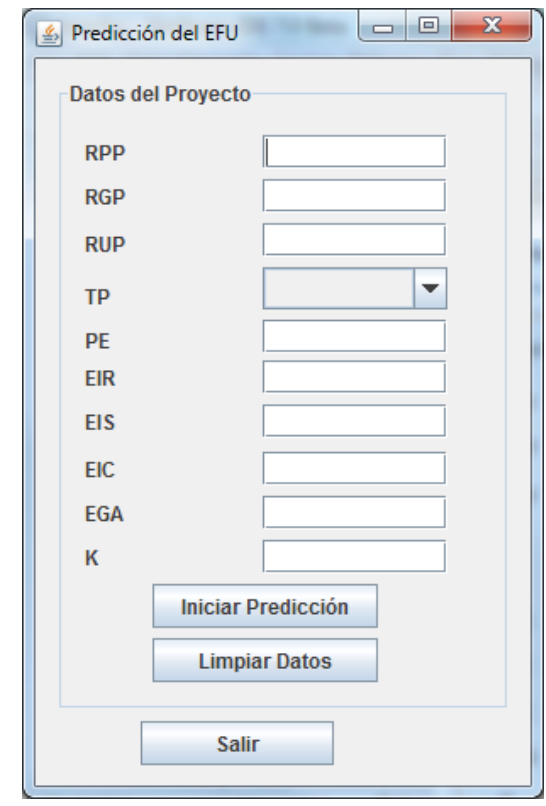

Figura 10. Interfaz visual

Fuente: Captura de pantalla de la interfaz elaborada.

El código del formulario (interfaz) se encuentra en el anexo 1.

\section{Conclusiones}

Este documento propone un modelo que ayuda al proceso de toma de decisiones en la selección de proyectos a ejecutar por las empresas constructoras, mediante un cálculo objetivo del indicador de efectividad y del cálculo de la probabilidad de éxito o fracaso del proyecto antes de su ejecución.

Se establece un proceso inteligente y predictivo en la gestión del riesgo de la empresa, en las fases de establecimiento de contexto, identificación y análisis del riesgo, que utiliza y evalúa los datos históricos de proyectos similares ejecutados por la organización, para identificar los factores de riesgo que afectan sus objetivos. 
Se establece un mecanismo para el diseño de indicadores históricos de medición, determinando los factores del riesgo que impactan en los objetivos del proyecto. Los indicadores reales son obtenidos sobre la base de las actuaciones reales de la organización.

Ayuda a mejorar el grado de confiabilidad en la toma de decisiones con factores de riesgo en las empresas constructoras, que podría servir para confirmar las predicciones subjetivas o realizar un replanteo de estas, proporcionando una manera de comparar los datos históricos sobre los riesgos identificados mediante el uso, como herramienta, de las redes neuronales artificiales.

\section{Bibliografía}

Apaza, Mario (2003). Balanced scorecard gerencia estratégica y del valor. Instituto de Investigaciones del Pacífico (ed.). Lima: Pacífico Editores.

Basili, Victor; Caldiera, Gianluigi y H. Dieter Rombach (1994). "The goal question metric approach", en Marciniak, John (ed.). Encyclopedia of software engineering, pp. 528-532. Nueva Jersey: John Wiley \& Sons, Inc.

Berardinis, Lawrence (1992). "Untangling the mystery of neural networks". Machine Design. Vol. 65, num. 13, pp. 55-59.

Cruz Lezama, Osaín (2007). Componentes de un proyecto de inversión. <http://www. cadperu.com>. [Consulta: 15 de julio del 2010].

Del Carpio, Javier y Roberto Eyzaguirre (2007). “Análisis de riesgo en la evaluación de alternativas de inversión utilizando Crystal Ball". Industrial Data. Vol.10, núm. 1, pp. 55-58. <http://www.scielo.org.pe/scielo.php?pid=S1810-99932007 000100009\&script=sci_arttext>. [Consulta: 20 de octubre del 2011].

Galarce, Yorka; Pomier, María y Carolina Pastén (2006). Gestión de riesgos. Seminario para optar al título profesional de contador público-contador auditor Arica: Universidad Arturo Prat, Departamento de Auditoría y Sistema de Información.

Instituto Nacional de Estadística e Informática (2011). "Indicadores de precios de la economía". INEI, Dirección Técnica de Indicadores Económicos, Dirección Ejecutiva de Indices.

Isasi, Pedro e Inés Galván (2004). Redes neuronales artificiales. Un enfoque práctico. Madrid: Pearson education S.A.

Kohonen, Teuvo (1982). "Analysis of a simple self-organizing process". Biological Cybernetics, 44, 135-140. Berlín: Springer. 
Koprinarov, Bratoy (2005). El riesgo empresarial y su gestión. Caracas: Analítica Consulting 1996. <http://www.analitica.com/va/economia/opinion/ 5753437.asp>. [Consulta: 31 de marzo del 2010].

Michie, Donald; Spiegelhalter, David y Charles Taylor (eds.) (1994). Machine learning, neural and statistical classification. Nueva York: Ellis Horwood.

Organismo Supervisor de Contrataciones del Estado (OSCE) (1 de febrero del 2009). Decreto Supremo 184-2008-EF, Reglamento de Ley de Contrataciones del Estado. OSCE, Dirección de Arbitraje Administrativo. Lima.

Rumelhart, David; Hinton, Geoffrey y Ronald Williams (1986). "Learning internal representations by error propagation", en Rumelhart, D. E., McClelland, J. L. y el PDP Research Group. Parallel distributed processing: Explorations in the microstructure of cognition. Vol. 1, Massachusetts: MIT Press.

Salgueiro, Amado (2001). Indicadores de gestión y cuadro de mando. Madrid: Díaz de Santos.

Sarcià, Salvatore A., Salvatore Alessandro Sarcià, Giovanni CantoneCantone, Giovanni; y Victor Basili Victor R. Basili (2007). "A statistical neural network framework for risk management process: from the proposal to its preliminary validation for efficiency". Ponencia presentada en International Conference on Software and Data Technologies, organizada por Icsoft. Barcelona, 25-57 de julio.

Sarle, Warren (2002). Neural networks FAQ. <ftp://ftp.sas.com/pub/neurall/FAQ. html>. [Consulta: 20 de septiembre del 2010].

Smith, J. Clarke (1990). A neural network-could it work for you?". Financial Executive. Año 2, vol. 6, núm. 3, pp. 26-30.

Standards Australia (1999). AS/NZS 4306:2004 Risk Management Standard. Sidney: Standards Australia International. 


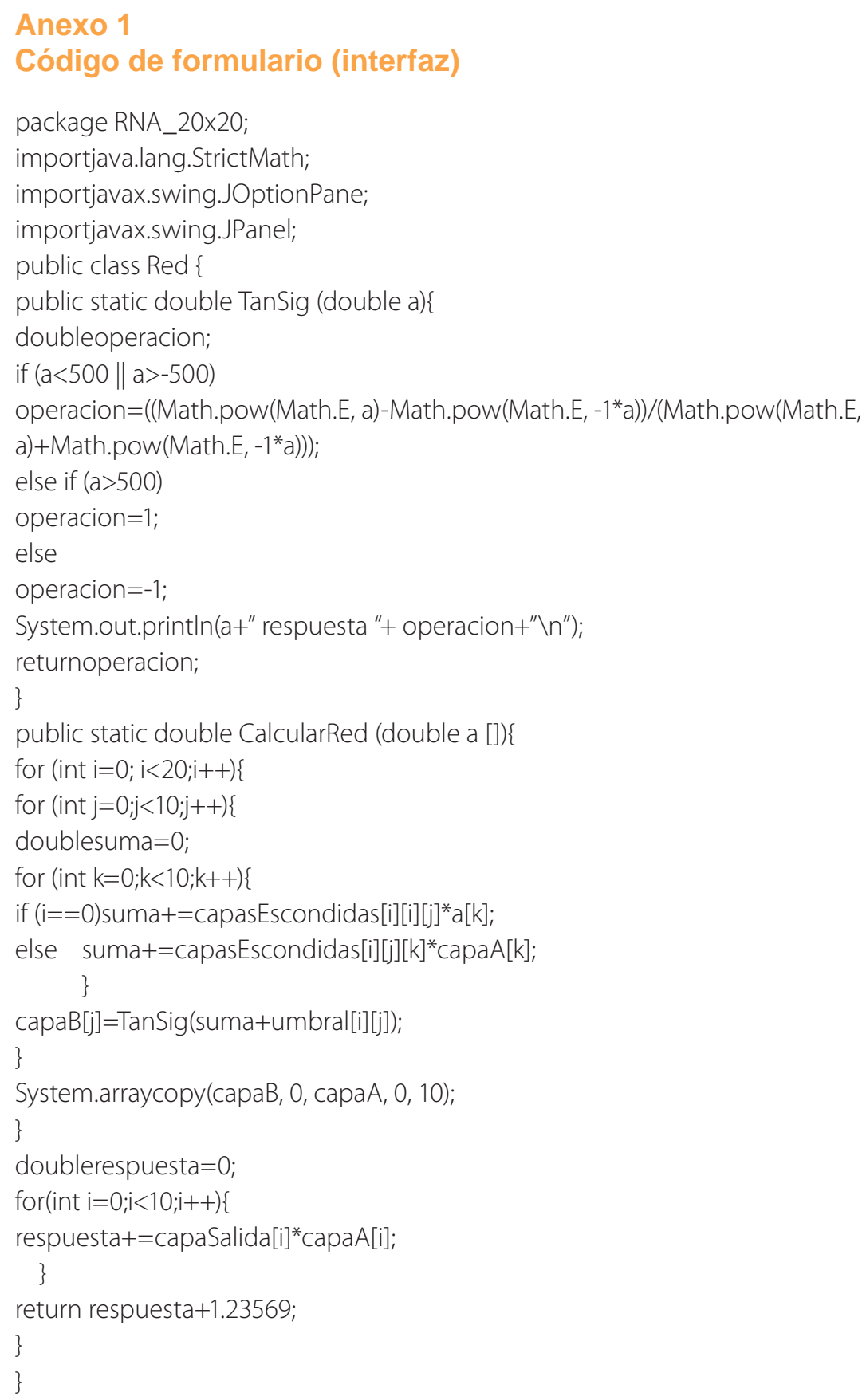




\section{Anexo 2:}

\section{Glosario de terminos}

A priori: Previo; independiente de la experiencia, expresa incertidumbre, no tiene en cuenta datos.

Activos: En relación con la seguridad de la información, se refiere a todo tipo de información o sistema relacionado con el tratamiento de esta que tenga valor para la organización.

Clasificación: Es la técnica que se utiliza para la identificación, agrupación y distribución sistemática de documentos o cosas semejantes, con características comunes o sistema determinado, y que con posterioridad pueden ser diferenciadas según su tipología fundamental. Dicho proceso se aplica de acuerdo a un esquema lógico predeterminado para señalar su ubicación. Cuando se trata de libros o documentos se llama clasificación bibliográfica o documental.

Control: Las políticas, los procedimientos, las prácticas y las estructuras organizativas concebidas para mantener los riesgos de seguridad de la información por debajo del nivel de riesgo asumido. Control también es utilizado como sinónimo de salvaguarda o contramedida.

Incidencia/Incidente: Evento único o serie de eventos de seguridad de la información inesperados o no deseados que poseen una probabilidad significativa de comprometer las operaciones de negocio y amenazar la seguridad de la información.

Isomorfa: Paralelismo estructural; la estructura de la representación corresponde, en menor o mayor grado, a la estructura de lo representado.

Neurotransmisores: Los neurotransmisores son las sustancias químicas que se encargan de la transmisión de las señales desde una neurona hasta la siguiente a través de las sinapsis. También se encuentran en la terminal axónica de las neuronas motoras, donde estimulan las fibras musculares para contraerlas.

Relación recursiva: Proceso mediante el cual una función se llama a sí misma en forma repetida, hasta que se satisface alguna determinada condición. El proceso se utiliza para computaciones repetidas, en las que cada acción se determina mediante un resultado anterior. Se pueden escribir de esta forma muchos problemas iterativos.

Sinapsis: La sinapsis es el proceso de comunicación neuronal, el lenguaje del sistema nervioso y la unión intercelular especializada entre neuronas. En estos contactos se lleva a cabo la transmisión del impulso nervioso. Este 
Redes neuronales para la optimización de la gestión del riesgo empresarial

se inicia con una descarga química que origina una corriente eléctrica en la membrana de la célula presináptica (célula emisora); una vez que este impulso nervioso alcanza el extremo del axón (la conexión con la otra célula), la propia neurona segrega un tipo de compuestos químicos (neurotransmisores) que se depositan en el espacio sináptico (espacio intermedio entre esta neurona transmisora y la neurona postsináptica o receptora). Estas sustancias segregadas o neurotransmisores (noradrenalina y acetilcolina, entre otros) son los encargados de excitar o inhibir la acción de la otra célula llamada célula postsináptica. 